\title{
Caracterização dos idosos internados por doença respiratória aguda em um hospital escola terciário
}

\author{
Characterization of elderly inpatient with acute respiratory disease \\ in a tertiary medical school hospital
}

\begin{abstract}
Vivian Romanholi Coria ${ }^{1}$, Ingrid Helen Grigolo ${ }^{2}$, Gabriela Schimidt Defende ${ }^{1}$, Verena Mattos Mutter $^{2}$, Luis Lemos Moras ${ }^{1}$, Stephanie Tairowite Morales ${ }^{3}$, Larissa Helena Marques Carrai ${ }^{2}$, Ana Laura de Oliveira Dias ${ }^{1}$, Nauyla Miranda da Costa ${ }^{4}$, Alexandre de Andrade Budin ${ }^{2}$, Camila Dias Rodrigues $^{3}$, Renata Borges Marchiori ${ }^{3}$, Danilo Tacinari Sasso ${ }^{1}$, Andre Gaiotto Calocini ${ }^{2}$, Juliana Cristina Lima ${ }^{3}$, Andréia Francesli Negri Reis ${ }^{5}$, Fátima Grisi Kuyumjiann ${ }^{6}$, João de Castilho Cação
\end{abstract}

Romanholi-Cória V, Grigolo IH, Defende GS, Mutter VM, Moras LL, Morales ST, Carrai LHM, Dias ALO, Costa NM, Budin AA, Rodrigues CD, Marchiori RB, Sasso DT, Calocini AG, Lima JC, Reis AFN, Kuyumjian, FG, Cação, JC. Caracterização dos idosos internados por doença respiratória aguda em um hospital escola terciário/ Characterization of elderly inpatient with acute respiratory disease in a tertiary medical school hospital. Rev Med (São Paulo). 2017 abr.-jun.;96(2):94-102.

RESUMO: Objetivo: Caracterizar o perfil sociodemográfico, clínico e de fatores de risco de idosos internados por Doença Respiratória Aguda (DRA) no Hospital de Base de São José do Rio Preto. Metodologia: Pesquisa quantitativa, descritiva e retrospectiva, com dados coletados através da análise de 94 prontuários médicos de idosos com idade igual ou superior a 60 anos, internados no período de agosto de 2012 a setembro de 2013 por DRA no Hospital de Base de São José do Rio Preto.
Resultados: O estudo identificou que entre os 94 idosos internados por DRA, 56,4\% eram homens $(\mathrm{n}=53)$, com 76 anos de idade ou mais $(55,3 \%)$, brancos $(88,3 \%)$, casados $(54,2 \%)$, com baixa escolaridade (19,1\% não alfabetizados e $53,3 \%$ com até 4 anos de estudo), portadores de pelo menos uma comorbidade (83\%), e apresentavam pelo menos um fator de risco para DRA como tabagismo e/ou desnutrição. Dos classificados com dependência funcional, a maioria eram idosos acima dos 75 anos. Quanto

Trabalho Apresentado, categoria ORAL - XXI Congresso Médico do Oeste Paulista, 18-20 set. 2014, na Sociedade de Medicina e Cirurgia de São José do Rio Preto.

Fonte de Financiamento: Ministérios da Saúde e da Educação (Programa de Educação pelo Trabalho para a Saúde - PET, uma estratégia do Programa Nacional de Reorientação da Formação Profissional em Saúde).

1. Acadêmica(o) de Medicina da Faculdade de Medicina de São José do Rio Preto-SP, Brasil. Emails: vromanholi@gmail.com, gabi_schde@hotmail. com, luislemossp@hotmail.com, analaura.odias@gmail.com, danilotacinari@gmail.com,

2. Médica(o) Graduada(o) pela Faculdade de Medicina de São José do Rio Preto-SP, Brasil. Email: ingridgrigolo@gmail.com, verena_mattos@hotmail. com, larihmc@gmail.com, alexandrebudin@hotmail.com, andre_gaiotto@hotmail.com, andre_gaiotto@hotmail.com.

3.Enfermeira Graduada pela Faculdade de Medicina de São José do Rio Preto-SP, Brasil. Email: stephanie morales433@hotmail.com, camilaenfermagemfamerp@outlook.com,re_marchiori@hotmail, cristina.ju@hotmail.com.

4. Enfermeira residente em do Programa Uniprofissional de Residência em Enfermagem Cardiovascular do Instituto Dante Pazzanese de Cardiologia São Paulo, Brasil. Email: nauylamc@outlook.com.

5. Preceptora do Programa de Educação pelo Trabalho - PET Saúde/Vigilância em Saúde e Gerente da Vigilância Epidemiológica Municipal de São José do Rio Preto, SP, Brasil. Email: andreiafrancesli@yahoo.com.br,

6. Preceptora do Programa de Educação pelo Trabalho - PET Saúde/Vigilância em Saúde e Odontologista do Grupo de Vigilância Epidemiológica de São José do Rio Preto GVE XXIX, São Paulo, Brasil. Email: fatimagk@globo.com.

7. Tutor do Programa de Educação pelo Trabalho - PET Saúde/Vigilância em Saúde e Médico Geriatra, Professor Doutor Adjunto do Departamento de Medicina I da Faculdade de Medicina de São José do Rio Preto, São Paulo, Brasil. Email: joaoccacao1020@gmail.com.

Endereço para correspondência: Vivian Romanholi Cória. Rua Teodoro Demonte, 88 Apto. 302. Vila São Manoel, São José do Rio Preto, SP, Brasil. CEP: 15091-260. 
à situação cognitiva, menos da metade dos idosos apresentava algum déficit relatado em prontuário, e entre estes a maioria era maior de 75 anos. Mais de $70 \%$ dos pacientes $(n=68)$ receberam alta com melhora, sendo que mais da metade $(n=38)$ tinham 75 anos de idade ou menos. Do total, 17 pacientes evoluíram a óbito, sendo que destes, $88,2 \%(n=15)$ tinham acima de 75 anos. Após a alta médica, um pequeno número de pacientes $(n=11)$ foram contra-referenciados às suas Unidades Básicas de Saúde de origem. Conclusão: Os resultados do estudo apontam para a importância de se reforçar as redes de cuidados voltadas aos idosos comprometidos por DRA, especialmente aqueles com idade avançada, fatores de risco presentes, comorbidades associadas, dependência funcional e déficit cognitivo, devido à alta probabilidade de má evolução. A capacitação dos profissionais de saúde dos diferentes níveis de atenção à saúde e a criação de estratégias para educação da população são ações fundamentais para a consolidação destas redes de cuidados e, consequentemente, auxiliar na prevenção e no diagnóstico precoce, visando à redução das internações e a mortalidade por DRA.

Descritores: Idoso; Hospitalização; Doenças respiratórias; Hospitais de ensino.

ABSTRACT: Objective: To characterize the sociodemographic and clinic profiles and risk factors of elderlies hospitalized by acute respiratory disease (ARD) in Hospital de Base de São José do Rio Preto. Metodology: A quantitative, descriptive and

\section{INTRODUÇÃO}

$\mathrm{O}$ rápido envelhecimento da população brasileira, iniciado mais intensamente a partir da década 70 do século passado, fruto da melhoria dos serviços de saúde, diminuição da fecundidade e aumento da expectativa de vida, resultou na mudança da composição etária da população brasileira, com crescimento progressivo da população idosa ou em vias de envelhecimento e diminuição da proporção de crianças e indivíduos jovens, a esse processo dá-se o nome de transição demográfica ${ }^{1,2}$. Tal processo gera a necessidade de se estruturar os serviços de saúde para melhor atender às necessidades da população, emergindo a necessidade da adoção de modelos de educação mais adequados às necessidades do perfil epidemiológico mais marcado por doenças crônicas do que por doenças infecciosas ${ }^{3}$.

No entanto, as doenças respiratórias agudas (DRAs) ainda constituem um importante problema de saúde pública mundial, especialmente na população idosa, devido à sua elevada incidência, ao custo para o sistema de saúde, às potenciais complicações em virtude das comorbidades, da dependência funcional, da redução de mobilidade e da cognição comprometida, bem como aos elevados índices de morbimortalidade observados em países subdesenvolvidos e em desenvolvimento ${ }^{1}$.

As DRAs abrangem os processos inflamatórios com duração de 1 a 21 dias que incidem no trato respiratório, podendo ser infecciosos (como a gripe, causada por vírus; e as pneumonias, geralmente bacterianas) ou não infecciosos retrospective study, with data collected through the analysis of 94 medical records of elderly inpatient, 60 years or older, hospitalized from August 2012 to September 2013 due to ARD. Results: This study identified that among 94 elderly inpatient with acute respiratory disease, major were men $(n=53), 76$ years old or more $(\mathrm{n}=52)$, white $(\mathrm{n}=83)$, and had at least one risk factor for ARDs, such as smoking and/or bad nutrition. Most of the elderly over 75 years were dependent on daily life activities. Less than a half had cognitive deficit reported in their medical records, and most of them were more than 75 years old. More than $70 \%$ of the patients were discharged with improvement (n $=68)$, and more than half were 75 years old or less $(n=38)$. Among the 94 patients, 17 have died, being $88.2 \%$ over 75 years old $(\mathrm{n}=15)$. After discharge, a small number of patients were referred to their Primary Care Unit $(\mathrm{n}=11)$. Conclusions: The results of this study appoint to the importance of increasing care networks for elderly people affected by ARD, especially the oldiest, with risk factors, associated comorbidities, functional dependence and cognitive deficit, due to the high probability of bad prognosis. Training professionals at different levels of health care and creation of strategies to instruct the population about ARD are fundamental actions, in order to consolidate these care networks and, consequently, support prevention and early diagnosis, targeting the reduction of hospitalizations and mortality due to ARD.

Keywords: Aged; Hospitalization; Respiratory tract diseases; Hospitals, teaching.

(como asma e rinite alérgica). As DRAs podem também ser resultado de um processo crônico agudizado. Suas principais manifestações clínicas são tosse, dificuldade respiratória, dor de garganta, corrimento nasal e dor de ouvido, no entanto, nem sempre é possível distinguir sua origem $^{4}$.

Uma das principais causas de hospitalização e de mortalidade entre as pessoas acima dos sessenta anos são as infecções respiratórias e suas complicações ${ }^{5}$. Dentre os idosos com Doença de Alzheimer, em pesquisa realizada no Canadá, as infecções respiratórias ocuparam o segundo lugar de causas que contribuíram para a morte dos pacientes ${ }^{6}$. A influenza e a pneumonia adquirida na comunidade são problemas de saúde pública ${ }^{7}$.

Os dados do Sistema de Informações sobre Mortalidade (SIM) do Ministério da Saúde ${ }^{8}$ demonstraram que mais de $60 \%$ do total de óbitos ocorridos em 2007 , entre idosos com idade entre 60 e 74 anos, residentes no Estado de São Paulo, eram considerados evitáveis e que, entre eles, o segundo grupo de doenças mais importantes foram as do trato respiratório, especialmente a pneumonia.

Alterações na fisiologia do homem senil proporcionam maior susceptibilidade à DRA, seja pela diminuição da eficiência imunológica, queda da atividade ciliar, elevação da rigidez pulmonar e outros fatores ${ }^{5}$. Nessa mesma faixa de idade é pertinente demonstrar que idosos do sexo masculino apresentam maior incidência 
de hospitalização por doenças respiratórias quando comparados às mulheres, seja por fatores culturais como a menor procura de atendimento de saúde, ou por hábitos de vida menos saudáveis, como tabagismo e maior consumo de álcool ${ }^{9}$.

Outra causa para o aumento das doenças respiratórias no grupo de idosos é a crescente urbanização. Estudos recentes comprovam que a poluição atmosférica nos grandes centros tem sido identificada como um problema de saúde pública, comprometendo ainda mais o sistema respiratório dos idosos, além das alterações características da senescência, aumentando os atendimentos em prontossocorros, as internações hospitalares e a mortalidade ${ }^{10}$.

$\mathrm{Na}$ tentativa de diminuir os agravos por doenças respiratórias no idoso, começou-se a implementar políticas públicas de vacinação, sobretudo a de influenza, disponível gratuitamente no Brasil desde 1999 ${ }^{11 .}$ Estudos mostraram queda da taxa de mortalidade nos dois anos posteriores às campanhas vacinais $(2000 \text { e 2001) })^{12}$. Posteriormente, um estudo realizado em 496 municípios gaúchos mostrou que aqueles que atingiram a meta de $80 \%$ de idosos vacinados ou mais apresentaram menor índice de mortalidade a cada mil idosos ${ }^{13}$.

$\mathrm{O}$ fato da população nacional com 65 anos ou mais elevar-se de $5,9 \%$ em 2000 a $7,4 \%$ em $2010^{8}$ e das infecções respiratórias e suas complicações estarem entre as principais causas de hospitalização/mortalidade nos idosos revelam a importância deste estudo para caracterizar o perfil dos idosos internados por DRA, visando às melhorias nas estratégias de reconhecimento dos idosos em risco de obterem piores desfechos como resultados da doença.

\section{OBJETIVOS}

Caracterizar o perfil sociodemográfico, clínico e de fatores de risco para internação de idosos por DRA no Hospital de Base de São José do Rio Preto.

\section{METODOLOGIA}

Trata-se de pesquisa quantitativa, descritiva e retrospectiva, realizada a partir da análise de dados de prontuário de 94 idosos, com 60 anos ou mais, internados com diagnóstico de DRA no Hospital de Base de São José do Rio Preto no período de agosto de 2012 a setembro de 2013, provenientes da área de abrangência do Distrito Escola III e IV deste município, tabulando-se as variáveis epidemiológicas e clínicas: gênero, idade, etnia, ocupação, escolaridade, estado conjugal, presença ou não de comorbidades, estratificação das principais comorbidades, presença ou não de fatores de risco para DRA, dependência funcional, estado cognitivo, condições de alta hospitalar/ desfecho e encaminhamento após a internação. Procuraramse também informações em prontuário sobre a situação vacinal dos pacientes.
Os itens avaliados para estimar o grau de dependência funcional foram: dificuldade para deglutição, capacidade para alimentar-se, tomar remédios e realizar cuidados de higiene sozinhos, sendo estas informações obtidas nas evoluções do prontuário médico, registradas durante a internação.

Para estimar o status cognitivo dos pacientes, foram utilizados dados da anamnese, exame físico geral e exame neurológico, sendo que o idoso consciente e orientado em tempo e espaço, capaz de prestar suas próprias informações foi considerado com a cognição adequada e aquele que apresentou alteração em qualquer um destes itens foi classificado como comprometido.

O estudo obteve aprovação do pelo Comitê de Ética em Pesquisa em Seres Humanos da Faculdade de Medicina de São José do Rio Preto, através do CAAE $\mathrm{n}^{\circ} 26680714.6 .0000 .5415$ e faz parte do projeto financiado pelos Ministérios da Saúde e da Educação, intitulado "Programa de Educação pelo Trabalho para a Saúde" (PET), uma estratégia do Programa Nacional de Reorientação da Formação Profissional em Saúde ${ }^{15}$, que visa à construção de novos cenários de prática e estimula alterações curriculares que coloquem os alunos em contato com as demandas e necessidades reais da população. A Faculdade de Medicina de São José do Rio Preto em parceria com a Secretaria Municipal de Saúde coordenou o PET "Avaliação e reorganização da Rede de Cuidado a Doença Respiratória Aguda (DRA) em Idosos", composto por 15 acadêmicos de Medicina e Enfermagem, um docente tutor e dois preceptores da rede de atenção básica.

A caracterização dos idosos internados obtida neste estudo serviu de base para construção de instrumento de avaliação que, em etapa posterior do projeto, foi aplicado aos profissionais e funcionários das Unidades Básicas de Saúde (UBSs) da área de abrangência do Distrito Escola III e IV de São José do Rio Preto, permitindo identificar o grau de conhecimento destes trabalhadores, bem como as deficiências, em relação ao atendimento das DRAs na rede de atenção básica. Com tais subsídios, a última etapa do projeto consistiu na realização de oficinas para capacitação sobre DRA aos profissionais da rede, objetivando a melhora da qualidade da rede de cuidados dos idosos da região, visando à diminuição das internações por DRA.

\section{RESULTADOS}

Os resultados mostraram que, do total de 94 prontuários analisados, $53(56,4 \%)$ eram de pacientes do gênero masculino e $41(43,6 \%)$ do gênero feminino, sendo $52(55,3 \%)$ pacientes com idade igual ou superior a 76 anos, sendo a média de idade de 76,8 anos. Quanto à etnia, 88 idosos $(88,3 \%)$ foram declarados brancos. No que se refere à ocupação, 30 eram aposentados $(31,9 \%)$, 29 eram "do lar" (30,85\%), 29 foram classificados como “outras" ocupações (30,85\%) e em 6 prontuários $(6,4 \%)$ não 
constava a informação. Ao analisar a escolaridade houve predomínio da categoria que teve até 4 anos de estudo, com 50 pacientes $(53,3 \%), 16$ pacientes $(17 \%)$ tinham de 4 a 8 anos de estudo e a minoria apresentava mais do que oito anos de estudo, correspondendo a 10 pacientes (10,6\%); 18 idosos $(19,1 \%)$ não eram alfabetizados. Em relação ao estado civil, 51 pacientes eram casados (54,2\%), 29 viúvos $(30,85 \%), 6$ solteiros $(6,4 \%), 7$ divorciados ou separados $(7,45 \%)$ e em 1 prontuário não constava a informação (Tabela 1).

Tabela 1. Perfil sociodemográfico dos idosos internados no Hospital de Base de São José do Rio Preto, no período de agosto de 2012 a setembro de 2013

\begin{tabular}{|c|c|c|}
\hline Varíável & $\mathbf{N}$ & $\%$ \\
\hline \multicolumn{3}{|l|}{ Gênero } \\
\hline Masculino & 53 & 56,4 \\
\hline Feminino & 41 & 43,6 \\
\hline \multicolumn{3}{|l|}{ Idade } \\
\hline 60 à 75 & 42 & 44,7 \\
\hline 76 ou mais & 52 & 55,3 \\
\hline \multicolumn{3}{|l|}{ Etnia } \\
\hline Branco & 83 & 88,3 \\
\hline Negro & 6 & 6,4 \\
\hline Outros & 5 & 5,3 \\
\hline \multicolumn{3}{|l|}{ Ocupação } \\
\hline Aposentados & 30 & 31,9 \\
\hline Do lar & 29 & 30,85 \\
\hline Outros & 29 & 30,85 \\
\hline Não Informado & 6 & 6,4 \\
\hline \multicolumn{3}{|l|}{ Escolaridade } \\
\hline Não alfabetizado & 18 & 19,1 \\
\hline Até 4 anos de estudo & 50 & 53,3 \\
\hline De 4 a 8 anos de estudo & 16 & 17,0 \\
\hline Acima de de 8 anos & 10 & 10,6 \\
\hline \multicolumn{3}{|l|}{ Situação Conjugal } \\
\hline Solteiros & 6 & 6,4 \\
\hline Casados & 51 & 54,2 \\
\hline Viúvos & 29 & 30,85 \\
\hline Divorciados/ separados & 7 & 7,45 \\
\hline Não informado & 1 & 1,1 \\
\hline
\end{tabular}

O estudo revelou que $44(46,8 \%)$ idosos internados por DRA apresentavam três ou mais comorbidades, 34 (36,2\%) apresentavam pelo menos uma doença além da DRA e 16 (17\%) dos idosos não possuíam ou desconheciam ter outra doença além da DRA.

Dentre as comorbidades analisadas, a hipertensão arterial foi a mais prevalente, com 50 idosos portadores $(53,2 \%)$, seguida por diabetes mellitus (24 pacientes) e doenças respiratórias crônicas (24 pacientes), cada uma representado $24,5 \%$ dos idosos; 33 idosos $(35,1 \%)$ apresentavam outras doenças cardiovasculares, 14 (14,9\%) doenças neurológicas e $9(9,6 \%)$ apresentavam neoplasias (Tabela 2).

Tabela 2. Principais comorbidades apresentadas pelos idosos, relatadas nos prontuários

\begin{tabular}{l|c}
\hline Comorbidades & $\mathbf{N}^{0}$ de pacientes \\
\hline Hipertensão & 50 \\
Diabetes Melitus & 24 \\
Doença Respiratória Crônica & 24 \\
Doenças Cardiovasculares & 33 \\
Doenças Neurológicas & 14 \\
Neoplasias & 9 \\
\hline
\end{tabular}

O presente estudo procurou, ainda, identificar nos prontuários a presença de fatores que aumentam o risco de DRA grave em idosos. Observou-se que 59,6\% $(n=56)$ dos idosos da amostra apresentavam um ou mais fatores de risco, sendo os mais prevalentes: 53 idosos com algum sinal de desnutrição $(56,3 \%)$ e 36 tabagistas $(38,3 \%)$.

A capacidade funcional dos pacientes e seus graus de dependência foram analisados considerandose a dificuldade para deglutir e a capacidade de se alimentar, higienizar e medicar sozinhos. Dos idosos cujas informações sobre estes itens constavam do prontuário, observou-se que havia maior número de idosos capazes de realizar as atividades na casuística, no entanto, tais dados não estavam informados (ou descritos claramente) em grande número de prontuários (Tabela 3 ).

Tabela 3. Caracterização da dependência funcional dos idosos internados de agosto de 2012 a setembro de 2013 por DRA, no Hospital de Base de São José do Rio Preto

\begin{tabular}{lccc}
\hline $\begin{array}{l}\text { Dependência } \\
\text { Funcional }\end{array}$ & Sim & Não & $\begin{array}{c}\text { Não } \\
\text { informado }\end{array}$ \\
\hline $\begin{array}{l}\text { Dificuldades de } \\
\text { deglutição }\end{array}$ & $26(27,7 \%)$ & $51(54,2 \%)$ & $17(18,1 \%)$ \\
Alimenta-se sozinho & $46(48,9 \%)$ & $24(25,5 \%)$ & $24(25,5 \%)$ \\
$\begin{array}{l}\text { Higieniza-se } \\
\text { independente }\end{array}$ & $35(37,2 \%)$ & $29(30,8 \%)$ & $30(31,9 \%)$ \\
$\begin{array}{l}\text { Toma remédios } \\
\text { sozinho }\end{array}$ & $39(41,5 \%)$ & $22(23,4 \%)$ & $33(35,1 \%)$ \\
\hline
\end{tabular}

Os pacientes que apresentaram dificuldade em qualquer um dos itens foram separados em dois grupos: até 75 e maiores de 75 anos de idade. A análise mostrou que mais de $75 \%$ dos idosos acima de 75 anos não se alimentavam, não se higienizavam ou não se medicavam sozinhos, além de $69,2 \%$ destes idosos apresentarem dificuldades para deglutir (Tabela 4). 
Romanholi-Cória V, et al. Caracterização dos idosos internados por doença respiratória aguda em um hospital.

Tabela 4. Dependência funcional dos idosos internados de agosto de 2012 a setembro de 2013 por DRA, no Hospital de Base de São José do Rio Preto, em relação à faixa etária

\begin{tabular}{lcc}
\hline Dependência funcional & $\begin{array}{c}\mathbf{N}^{\mathbf{0}} \text { idosos até } \\
\mathbf{7 5} \text { anos }\end{array}$ & $\begin{array}{c}\mathbf{N}^{\mathbf{0}} \text { idosos }>\mathbf{7 5} \\
\text { anos }\end{array}$ \\
\hline $\begin{array}{l}\text { Dificuldade deglutição } \\
\text { Não se alimenta }\end{array}$ & $8(30,8 \%)$ & $18(69,2 \%)$ \\
$\begin{array}{l}\text { sozinho } \\
\text { Não se higieniza } \\
\text { sozinho }\end{array}$ & $5(20,8 \%)$ & $19(79,2 \%)$ \\
Não se medica sozinho & $7(24,1 \%)$ & $22(75,9 \%)$ \\
\hline
\end{tabular}

A situação cognitiva dos pacientes, obtida através de dados da anamnese, exame físico geral e exame neurológico, apresentou resultados de acordo com a Tabela 5. Os resultados mostraram que dos 55 pacientes que mantinham uma situação cognitiva adequada $(58,5 \%$ do total de indivíduos do estudo), mais de $60 \%$ tinham até 75 anos. Já entre os pacientes cujo prontuário permitiu identificar algum comprometimento da cognição, $88 \%$ eram de idosos acima dos 75 anos.

Tabela 5. Situação cognitiva dos idosos internados de agosto de 2012 a setembro de 2013 por DRA, no Hospital de Base de São José do Rio Preto, de acordo com o prontuário médico

\begin{tabular}{llll}
\hline $\begin{array}{l}\text { Situação } \\
\text { cognitiva }\end{array}$ & $\begin{array}{l}\text { Até 75 } \\
\text { anos }\end{array}$ & $\begin{array}{l}\text { Acima de } \\
\mathbf{7 5} \text { anos }\end{array}$ & Total \\
\hline Adequada & $34(61,8 \%)$ & $21(38,2 \%)$ & $55(100 \%)$ \\
Comprometida & $3(12 \%)$ & $22(88 \%)$ & $25(100 \%)$ \\
Não Informado & $5(35,7 \%)$ & $9(64,3 \%)$ & $14(100 \%)$ \\
\hline
\end{tabular}

Em relação à s condições clínicas do paciente no ato da alta médica, 68 pacientes, o que representa $72,3 \%$ do total, recebeu alta em melhores condições clínicas em relação à admissão. Destes, a maioria $(55,9 \%)$ tinha até 75 anos. Cinco pacientes receberam alta hospitalar mais debilitados do que se encontravam antes da internação, estando 4 deles acima dos 75 anos, e 17 pacientes foram a óbito. Destes, 15 eram maiores de 75 anos de idade, representando $88,2 \%$ do total de mortes (Tabelas 6 e 7).

Tabela 6. Condições de alta dos idosos internados de agosto de 2012 a setembro de 2013 por DRA, no Hospital de Base de São José do Rio Preto, de acordo com o prontuário médico

\begin{tabular}{lc}
\hline Condições de Alta & $\begin{array}{c}\mathbf{N}^{\mathbf{0}} \text { de Pacientes } \\
\text { (do total de } 94 \text { idosos) }\end{array}$ \\
\hline Melhorado & $68(72,3 \%)$ \\
Piorado & $5(5,3 \%)$ \\
Óbito & $17(18,1 \%)$ \\
Não Informados & $4(4,3 \%)$ \\
\hline
\end{tabular}

Tabela 7. Condições de alta dos idosos internados de agosto de 2012 a setembro de 2013 por DRA, no Hospital de Base de São José do Rio Preto, de acordo com a faixa etária

\begin{tabular}{llll}
\hline $\begin{array}{l}\text { Condições } \\
\text { de Alta }\end{array}$ & $\begin{array}{l}\mathbf{N}^{0} \text { idosos até } \\
\mathbf{7 5} \text { anos }\end{array}$ & $\begin{array}{l}\mathbf{N}^{0} \text { idosos } \\
>\mathbf{7 5} \text { anos }\end{array}$ & $\begin{array}{l}\text { Total de } \\
\text { Idosos }\end{array}$ \\
\hline Melhorado & $38(55,9 \%)$ & $30(44,1 \%)$ & $68(100 \%)$ \\
Piorado & $1(20 \%)$ & $4(80 \%)$ & $5(100 \%)$ \\
Óbito & $2(11,8 \%)$ & $15(88,2 \%)$ & $17(100 \%)$ \\
\hline
\end{tabular}

Após a alta hospitalar, 11 idosos (11,7\% do total de idosos do estudo) receberam encaminhamento para suas Unidades de Saúde de origem; 1 paciente foi transferido para uma Instituição de Longa Permanência do Idoso (ILPI), $27(28,7 \%)$ foram encaminhados ao ambulatório próprio do hospital para acompanhamento nas especialidades e em 38 prontuários (40,4\%) não constavam informações de destino após alta hospitalar (Tabela 8).

É importante destacar que, nos dados de prontuário, não estava relatada a situação vacinal dos pacientes.

Tabela 8. Encaminhamento dos idosos após a internação

\begin{tabular}{l|l}
\hline Encaminhamento após a internação & \multicolumn{1}{l}{$\mathbf{N}^{\mathbf{0}}$ de Pacientes } \\
\hline Unidade Básica & $11(11,7 \%)$ \\
ILPI & $1(1,1 \%)$ \\
Ambulatório & $27(28,7 \%)$ \\
Procedimentos após Óbito & $17(18,1 \%)$ \\
Não Informado & $38(40,4 \%)$ \\
\hline
\end{tabular}

\section{DISCUSSÃO}

Os resultados obtidos no estudo se encontram em consonância com os obtidos em trabalhos realizados com idosos internados em hospitais de outros serviços, segundo variáveis clínicas e sociodemográficas, reforçando que os homens são mais susceptíveis a internações por DRA ${ }^{16,17,18}$. As doenças do aparelho cardiovascular e do aparelho respiratório são as causas mais comuns de hospitalização dos idosos do sexo masculino entre a sétima e a oitava décadas de vida ${ }^{16-19}$. A discussão sobre maior prevalência de tais agravos nos homens é centrada nas hipóteses de que isso se deva a fatores culturais, como uma menor afinidade deles com os serviços de saúde, hábitos menos saudáveis assumidos ao longo da vida (tabagismo, etilismo, nutrição inadequada, falta de acompanhamento médico constante), menor aderência às práticas de autocuidado, maior exposição à prática de violência, muito embora este item seja mais comum entre os homens mais jovens. 
Alguns estudos, por outro lado, mostram maior número de idosas em situação de hospitalização, no entanto, as causas mais frequentes em tais estudos são os traumas e as quedas ${ }^{20,21,22}$. Talvez a menor incidência de osteopenia/ osteoporose nos homens, além de maior habilidade com atividades que exigem esforço físico ao longo da vida, possam explicar o porquê dos homens estarem menos susceptíveis aos traumas e quedas.

No que diz respeito à idade, os estudos apontam uma maior frequência de hospitalizações na oitava década de vida $^{23,24,25,26}$, confirmando-se os achados do presente estudo. Um fato interessante é que nas casuísticas encontradas nas quais a média de idade é abaixo dos 70 anos, predominam os homens e as doenças cardiovasculares e do sistema respiratório ${ }^{16,18}$, enquanto que naquelas em que a faixa etária média de idosos internados está acima dos 80 anos, predomina o sexo feminino ${ }^{21,27}$, internadas por trauma ${ }^{21}$. Os homens, em geral, apresentam menor expectativa de vida de vida em relação às mulheres e, conforme já exposto, isto pode ser explicado pelos hábitos menos saudáveis durante a vida, como o tabagismo e a má alimentação, o que pode explicar as internações mais precoces e suas principais causas.

Houve predomínio de indivíduos que se declararam de cor branca neste estudo, enquanto que no estudo conduzido por Coutinho et al. ${ }^{21}$, no Ceará (Nordeste) predominaram os pardos. As características da colonização do Brasil podem explicar tais resultados, visto que no Sudeste houve predomínio de europeus e, já no Nordeste, há maior índice de negros e, consequentemente, maior miscigenação de cores.

O perfil de idosos com baixa escolaridade, predominando indivíduos com até 4 anos de estudos, vai de encontro ao perfil encontrado em outros estudos brasileiros semelhantes ${ }^{17,20,24,25}$, Este fator pode explicar a dificuldade de entendimento das orientações fornecidas pelos profissionais de saúde e de acesso adequado a cuidados preventivos ${ }^{23}$. A análise dos dados demonstrou, também, que nesta casuística a maioria dos idosos eram casados. Acredita-se que as relações afetivas mais estáveis e duradouras, favoreçam a assistência, pela possibilidade do parceiro representar um cuidador, permitindo, assim, o alcance da longevidade ${ }^{23}$.

Diversos estudos anteriores apontam a hipertensão e a diabetes como principais comorbidades presentes nos idosos, que muitas vezes estão associadas à causa principal da internação $0^{21,23,25,26}$. A presença destas comorbidades na população do presente estudo foi relevante, indo de acordo ao cenário mundial marcado por tais doenças. É importante ressaltar a existência do Hiperdia, destinado ao cadastramento e acompanhamento de portadores de hipertensão arterial e/ou diabetes mellitus atendidos na rede ambulatorial do Sistema Único de Saúde - SUS, permitindo gerar informação para aquisição, dispensação e distribuição de medicamentos de forma regular e sistemática a todos os pacientes cadastrados ${ }^{28}$. No entanto, na prática o que se encontra é um sistema que ainda não se apresenta capaz de rastrear, cadastrar e acompanhar todos estes doentes, especialmente devido à quantidade de trabalhadores/ prestadores de serviços insuficientes para atender às suas regiões específicas.

O presente estudo procurou, ainda, identificar nos prontuários a presença de fatores que aumentam o risco de DRA grave em idosos. Observou-se que quase $60 \%$ dos idosos da amostra apresentavam um ou mais fatores de risco, sendo que mais da metade da população idosa internada por DRA apresentava algum sinal de desnutrição, normalmente associada às doenças consuptivas, como as neoplasias, e quase $40 \%$ eram tabagistas, que pode levar a alterações estruturais no pulmão (Doença Pulmonar Obstrutiva Crônica) e predispor a infecções respiratórias ${ }^{29}$. Um estudo realizado em um Hospital Universitário em São Paulo destaca a desnutrição como um fator de risco independente para morte hospitalar ${ }^{27}$.

Dentre os prontuários em que constavam informações sobre atividades como deglutição, capacidade de alimentarse, higienizar-se e tomar remédio sozinho, extraiu-se que a maioria dos idosos era independente para estas atividades, e os índices de maior dependência estavam entre os maiores de 75 anos. Idosos nesta situação são mais vulneráveis a delirium e infecções nosocomiais o que pode representar importante fator de risco para má evolução ${ }^{27}$. Em relação às falhas da cognição, foram detectadas em 25 prontuários apenas, sendo que quase $90 \%$ destes estavam acima de 75 anos. Avelino et al. ${ }^{27}$ também destacam que entre os idosos que vieram a óbito em seu estudo, a maioria apresentou nível significativamente maior de dependência funcional e declínio cognitivo em relação aos que receberam alta hospitalar. Faz-se necessário observar que os itens dependência funcional e perda cognitiva dos pacientes de nosso estudo podem estar subestimados, visto que foram inferidos à partir de dados do prontuário.

Ademais, objetivou-se, neste estudo, identificaramse determinantes de desfechos desfavoráveis (óbitos), e observou-se que, entre os idosos que faleceram durante a internação, 88,2\% tinham mais de 75 anos. Em função disso, procurou-se detectar quais fatores agregados à idade mais elevada poderiam ter determinado a má evolução - a incapacidade funcional foi relacionada ao pior prognóstico. Os dados são concordantes com os estudos disponíveis na literatura que mostram associação direta da pior capacidade funcional, principalmente restrição ao leito e alteração da deglutição, com predisposição a pneumonia e pior prognóstico ${ }^{30,31}$. Não são evidentes dados diretos ligando estado cognitivo a desfecho desfavorável de DRA, mas provavelmente em consequência do comprometimento global que acontece nas demências avançadas, a DRA é um dos principais eventos determinantes de morte nesses indivíduos.

Sobre o encaminhamento após alta, verificou- 
se nos prontuários relato de encaminhamento para acompanhamento na atenção básica em apenas 11 casos. Outros 27 pacientes foram encaminhados ao ambulatório do próprio hospital e em 38 prontuários não estava disponível a informação.

Uma parcela significativa dos idosos que recebem alta após uma DRA poderia se beneficiar de várias estratégias de atendimento oferecido na rede básica como: Serviço de Assistência Domiciliária (SAD), Unidade Básica da Saúde da Família (UBSF), além do seguimento regular em UBS, através da utilização dos serviços de contra-referência. Os dados apontam a necessidade de maior interação entre a rede terciária e primária, posto que o atendimento integrado seja sabidamente mais efetivo e barato. Após um evento agudo grave em idoso é importante o acompanhamento continuo oferecido na atenção primária, uma vez que os mesmos fatores que ocasionaram a DRA ainda podem estar presentes e devem ser gerenciados visando à diminuição das vulnerabilidades e taxas de internações no futuro ${ }^{32}$.

Queiroz et al. ${ }^{26}$ verificou em seu estudo que a maioria dos idosos recebeu alta hospitalar por melhora e, destes, a maior parte foi encaminhada para Unidades de Saúde da Família, o que demonstra o início da conscientização sobre a necessidade de acompanhamento constante dos idosos, especialmente após internação e, também, por suas doenças pré-existentes.

Vale ressaltar que o acompanhamento na atenção primária garante o acesso às vacinas necessárias para cada faixa etária, sendo a vacina contra as cepas do vírus influenza essencial na terceira idade, além da atualização da carteira de acordo com as demais comorbidades. A vacinação contra influenza ganhou muita importância nas últimas décadas, sendo que estudos da década de 90 , de importantes periódicos, já apontavam para a efetividade da vacina na redução das taxas de hospitalização e morte ${ }^{33}$. Um estudo norte- americano mostrou redução significativa do risco de hospitalização por influenza confirmada laboratorialmente entre os adultos com idade $\geq 50$ anos, independentemente da faixa etária ${ }^{34}$.

Por fim, dada a importância dos impactos sociais e financeiros das DRA na saúde pública, os achados do presente estudo e de suas etapas posteriores são fundamentais para ampliar a visibilidade em relação à saúde do idoso, bem como as demandas na busca incessante pelo aprimoramento da qualidade de vida. A capacitação dos trabalhadores da área da saúde é um dos passos essenciais para garantir a promoção à saúde, a detecção precoce de riscos, a prevenção de agravos e o diagnóstico precoce, evitando-se as complicações e favorecendo-se o envelhecimento saudável.

\section{CONCLUSÃO}

O estudo do perfil populacional com vulnerabilidade à DRA é fundamental para determinar fatores que podem orientar as estratégias para realização de ações que minimizem os riscos de agravos à saúde, além de aumentar a efetividade de medidas preventivas e terapêuticas, o que envolve a capacitação constante e atualização dos funcionários dos serviços de saúde, com diferentes níveis de formação, e a educação da população. Os dados depreendidos deste estudo apontam para a necessidade de organização de uma rede de cuidados especiais para idosos com idade avançada (> 75anos), com baixa escolaridade, portadores de comorbidades, desnutrição e tabagistas, dependentes funcionais e com déficit cognitivo, posto que é alta a probabilidade de má evolução.

\section{Limitações do estudo}

O estudo apresentou limitações dignas de nota.

A primeira limitação reside no próprio fato dos dados serem obtidos através da análise de prontuários, cujo preenchimento é feito por diversos profissionais, de diferentes áreas, com enfoque clínico distinto e interpretações de achados examinador-dependentes diversas. Além disso, nem todas as variáveis analisadas no estudo estavam disponíveis em todos os prontuários.

Outra limitação importante decorre da multiplicidade de estudantes envolvidos na coleta de dados de prontuário. Por mais que este documento exija objetividade para que os indivíduos que o acessem tenham a mais uniforme interpretação, a análise de cada aluno pode variar, de acordo com a experiência e com a capacidade de inferir observações através dos dados.

Por fim, por se tratar de uma população muito específica, limitada a uma região do Estado de São Paulo, torna-se limitada a extrapolação dos resultados obtidos para a população em geral, no entanto, as informações obtidas podem ser valiosas para o planejamento de ações viáveis.

Ética: Não há conflito de interesses entre os autores. Os autores também confirmam que este artigo é único e não está sob consideração ou publicado em qualquer outro periódico.

Declaração de Responsabilidade: Os autores do manuscrito intitulado "Caracterização dos idosos internados por doença respiratória aguda em um hospital escola terciário" declaram que: 1. Este manuscrito representa um trabalho original cujo conteúdo integral ou parcial não foi por nós submetido a outro periódico ou outra forma de publicação, seja no formato impresso ou eletrônico; 2 . Houve participação efetiva de todos os autores relacionados no trabalho, tornando pública sua responsabilidade pelo conteúdo apresentado; 3 . Não há qualquer conflito de interesse dos autores em relação a este manuscrito. 


\section{REFERÊNCIAS}

1. Chaimowicz F. A saúde dos idosos brasileiros às vésperas do século XXI: problemas, projeções e alternativas. Rev Saúde Pública. 1997;31:184-200. http://dx.doi.org/10.1590/S003489101997000200014.

2. Moreira MM. O envelhecimento da população brasileira em nível regional: 1940-2050. Anais do XI Encontro Nacional de Estudos Populacionais da Associação Brasileira de Estudos Populacionais, Caxambu, ABEP; 1998. v.3, p.103-24.

3. Cunha ACNP, Cunha NNP, Barnosa, MT. Geriatric teaching in Brazilian medical schools in 2013 and considerations regarding adjustment to demographic and epidemiological transition. Rev Assoc Med Bras. 2016;62(2):179-83. http:// dx.doi.org/10.1590/1806-9282.62.02.179.

4. Façanha MC, Pinheiro AC. Doenças respiratórias agudas em serviços de saúde entre 1996 e 2001, Fortaleza, CE. Rev Saúde Pública. 2004;38(3):346-50. http://dx.doi.org/10.1590/ S0034-89102004000300002.

5. França ISX, Marinho DDT, Baptista RS. Infecções respiratórias em idosos e vacinação anti-influenza: índices de morbimortalidade. Rev Rene Fortaleza. 2008;9(3):52-61.

6. Park J. Mortality from Alzheimer's disease in Canada: a multiple-cause-of-death analysis, 2004 to 2011. Statistics Canada - Health Reports. 2015;27(5):17-21.

7. Augusto DK, Miranda LFJR, Cruz CEG, Pedroso ERP. Estudo comparativo entre pacientes idosos internados com diagnóstico clínico de pneumonia comunitária, com ou sem confirmação radiológica. J Bras Pneumol. 2007;33(3):270-4. http://dx.doi.org/10.1590/S1806-37132007000300007.

8. DATASUS. Óbitos por causas evitáveis [citado 29 jan. 2013]. Disponível em: http://tabnet.datasus.gov.br/cgi/tabcgi. exe?sim/cnv/evitb10sp.def.

9. Furtado MA, Silva RH, Zaia JE, Nascif-Júnior IA. Influence of vaccination against influenza in elderly persons in the epidemiology of hospitalization for pneumonia. Investigação. 2011;11:17-23. Disponível em: ttp://publicacoes.unifran.br/ index.php/investigacao/article/view/499/402.

10. Braga ALF, Pereira LAA, Procópio M, André PA, Saldiva PHN. Associação entre poluição atmosférica e doenças respiratórias e cardiovasculares na cidade de Itabira, Minas Gerais, Brasil. Cad Saúde Pública. 2007;23(S4):570-8. http:// dx.doi.org/10.1590/S0102-311X2007001600017.

11. Donalisio MR, Ruiz T, Cordeiro R. Fatores associados à vacinação contra influenza em idosos em município do Sudeste do Brasil. Rev Saúde Pública. 2006;40(1):115-9. http://dx.doi.org/10.1590/S0034-89102006000100018.

12. Donalisio MR, Francisco PMSB, Latorre, Oliveira MRD. Tendência da mortalidade por doenças respiratórias em idosos antes e depois das campanhas de vacinação contra influenza no Estado de São Paulo: 1980 a 2004. Rev Bras Epidemiol. 2006;9(1):32-41. http://dx.doi.org/10.1590/S1415790X2006000100006

13. Bos AJG, Mirandola AR. Cobertura vacinal está relacionada à menor mortalidade por doenças respiratórias. Ciênc Saúde Coletiva. 2013;18(5):1459-62. http://dx.doi.org/10.1590/ S1413-81232013000500031.

14. Instituto Brasileiro de Geografia e Estatística. Primeiros resultados definitivos do Censo 2010: população do Brasil é de 190.755.799 pessoas [citado 30 nov. 2013]. Disponível em http://www.censo2010.ibge.gov.br.

15. Brasil. Ministérios da Saúde e da Educação do Brasil. Programa Nacional de Reorientação da Formação Profissional em Saúde - Pró-Saúde: objetivos, implementação e desenvolvimento potencial. Brasília, DF: Ministério da Saúde; 2007. (Série C. Projetos, Programas e Relatórios).

16. Santos MIPO. Perfil dos idosos internados no hospital geral em Belém (Pará). Esc Anna Nery R Enferm. 2007;11(1):23 -9 .

17. Storti LB, Fabrício-Whebe SCC, Kusumota L, Rodrigues RAP, Marques S. Fragilidade de idosos internados na clínica médica da unidade de emergência de um hospital geral terciário. Texto Contexto Enferm, Florianópolis. 2013;22(2):452-9.

18. Cunha BSS, Nascimento AS, Chaves SP. Perfil clínico e sociodemográfico de internação de idosos na unidade de emergência de um hospital geral. Estud Interdiscipl Envelhec (Porto Alegre). 2014;19(1):189-200.

19. Pinheiro FM, Santo FHE, Chibante CLP, et al. Perfil de idosos hospitalizados segundo Viginia Henderson: contribuições para o cuidado em enfermagem. Care Online. 2016;8(3):4789-95. doi: http://dx.doi. org/10.9789/21755361.2016.v8i3.4789-4795.

20. Paula FL, Fonseca MJM, Oliveira RVC, Rozenfeld S. Perfil de idosos com internação por quedas nos hospitais públicos de Niterói (RJ). Rev Bras Epidemiol. 2010;13(4):587-95.

21. Coutinho MLN, Samúdio MA, Andrade LM, Coutinho RN, Silva DMA. Perfil sociodemográfico e processo de hospitalização de idosos atendidos em um hospital de emergências. Rev Rene. 2015;16(6):908-1005. doi: 10.15253/2175-6783.2015000600018.

22. Rodrigues J, Mantovani MF, Ciosak SI. O idoso e trauma: perfil e fatores desencadeantes. J Nurs UFPE (Recife). 2015:9(2):7071-7. doi: 10.5205/reuol.7505-65182-1RV.0903201508.

23. Nascimento WO, Santos AMR, Ribeiro IP, Oliveira ADS. Perfil do idoso com insuficiência cardíaca internado em um hospital de urgência. Cogitare Enferm. 2016;21(4):1-10.

24. Rocha FCV, Paz LI, Nery NKB, Almeida GMB, Rocha LPV, Carvalho ML. Profile of elderly patients admitted to the hospital emergency. Rev Enferm UFPI. 2014;3(3):32-8.

25. Antunes JFS, Okuno MFP, Lopes MCBT, Campanharo CRV, Batista REA. Avaliação da fragilidade de idosos internados em serviço de emergência de um hospital universitário Cássia. Cogitare Enferm. 2015;20(2):266-73.

26. Queiroz DB, Oliveira LC, Araúlo CM, Reis LA. Perfil de internações de idosos em uma clínica de neurociências de 
um hospital público. Rev Enfermagem Contemporânea. 2016;5(1):16-24. doi: 10.17267/2317-3378rec.v5i1.441.

27. Avelino-Silva TJ, Farfel JM, Curiati JAE, Amaral JRG, Campora F, Jacob-Filho W. Comprehensive geriatric assessment predictsmortality and adverse outcomes in hospitalized older adults. BMC Geriatrics. 2014;14:129. Disponível em: http://www.biomedcentral.com/14712318/14/129.

28. DATASUS. Departamento de Informática do SUS. Hiperdia sistema de cadastramento e acompanhamento de hipertensos e diabéticos. Disponível em: http://datasus.saude.gov.br/ sistemas-e-aplicativos/epidemiologicos/hiperdia

29. Lima-Costa MFF, Guerra HL, Barreto SM, Guimarães RM. Diagnóstico de saúde da população idosa brasileira: Um estudo da mortalidade e das internações hospitalares públicas. Informe Epidemiol SUS. 2000;9:23-41. http:// dx.doi.org/10.5123/S0104-16732000000100003.

30. Lima-Costa MF, Barreto SM, Giatti, L. Condições de saúde, capacidade funcional, uso de serviços de saúde e gastos com medicamentos da população idosa brasileira: um estudo descritivo baseado na Pesquisa Nacional por Amostra de

Recebido em: 07.09.16

Aceito em: 24.04.17
Domicílios. Cad Saúde Pública. 2003;19(3):735-43. http:// dx.doi.org/10.1590/S0102-311X2003000300006.

31. Francisco PMSB, Donalisio MRC, Lattore MRDO. Tendência da mortalidade por doenças respiratórias em idosos do Estado de São Paulo, 1980 a 1998. Rev Saúde Pública 2003;37(2):191-6. http://dx.doi.org/10.1590/ S0034-89102003000200005.

32. Freitas EV, Py L, Neri, AL, Cançado FAXC, Gorzoni ML, Doll, J. Tratado de geriatria e gerontologia. 3a ed. São Paulo: Grupo Editorial Nacional (GEN); 2011

33. Nichol KL, et al. The efficacy and cost effectiveness of vaccination against influenza among elderly persons living in the community. N Engl J Med. 1994;778-84. http://www. nejm.org/doi/pdf/10.1056/NEJM199409223311206

34. Havers FP, Sokolow L, Shay DK, Farley MM, Monroe M, Meek J, Kirley PD, Bennett NM, et al. Case-control study of vaccine effectiveness in preventing laboratory-confirmed influenza hospitalizations in older adults, United States, 2010-11. Clin Infect Dis. 2016;pii:ciw512. http://cid. oxfordjournals.org/content/early/2016/08/01/cid.ciw512. 